\title{
Dynamic characteristics of pilot boards of structures
}

\author{
Khorisboy Alimov", Zakiryo Buzrukov, and Mirzohid Turgunpulatov \\ Namangan Engineering Construction Institute, Namangan, Uzbekistan
}

\begin{abstract}
This work is devoted to studying the issues of vibrations of pile foundations, taking into account soil conditions for various types of fastening of piles to structures and the development of rational methods for choosing the parameters of piles and their number in earthquake-resistant construction. Based on the results of theoretical studies, resonance curves were constructed for three types of fastening of the "structure-group of piles" system. A special technique was proposed, and a series of field tests were carried out to determine the amplitude-frequency characteristics of the system.
\end{abstract}

\section{Introduction}

Structures erected in seismic areas must, on the one hand, meet the requirements of seismic resistance; on the other hand, meet all the requirements for them in non-seismic areas. Thus, the design of structures for seismic areas is always challenging.

The main problem is studying the joint work of a building on subsoil foundations during seismic impacts. One of the main factors in this is the condition for filling a building with soil foundations. The transition to industrial methods of construction of residential, public and industrial buildings and engineering structures has contributed to the widespread use of pile foundations in recent years.

By transferring the load to deeper and more durable soils, they provide the structure with greater uniformity of settlement and, as a result, the absence of primary stresses in structural elements associated with uneven settlement.

Pile foundations have been widely used in housing construction in recent years. When constructing the foundations of high-rise buildings, several features arise, considering the engineering and geological conditions that must be taken into account when designing. Because the territory of our country is located in an earthquake-prone area, it is important to comply with special requirements for the design and construction of foundations for high-rise buildings. During construction in Tashkent City, the soil layer in this area was not dense enough, so it had to be strengthened. To strengthen the soil throughout the entire area of the construction site, soil piles with a diameter of $800 \mathrm{~mm}$ were used.

In this regard, it became necessary, using the experience of the work carried out, to develop a calculation method and propose a method for testing a building and structure on a

\footnotetext{
*Corresponding author: xorisboyalimov@gmail.com
} 
pile foundation under horizontal seismic impact as the most appropriate to the real operating conditions of buildings and structures on pile foundations during earthquakes.

This article's main purpose is to present the main results of experimental and theoretical studies and show the effectiveness of the use of pile foundations under dynamic loading.

The object of research was selected buildings and structures on pile foundations under construction in areas with partially arising seismic impacts. The object of research was studied experimentally and theoretically. For a theoretical study, an analytical solution to the problem was selected, and for experimental studies, a six-component seismic platform was selected, which was built on pile foundations.

The seismic platform has a plan size of $13 \times 13 \times 6 \mathrm{~m}$, a foundation shaft size of $8 \times 8 \times$ $3.5 \mathrm{~m}$, a volume of $800 \mathrm{~m} 3$, and a weight of 2000 tons (Figure 1).

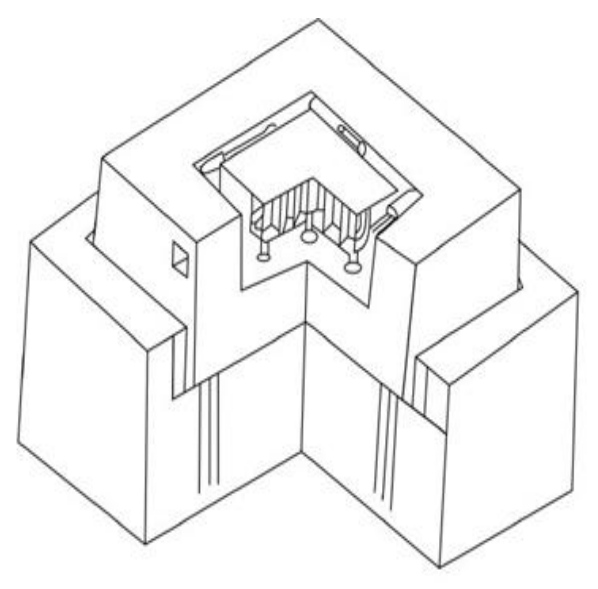

a)

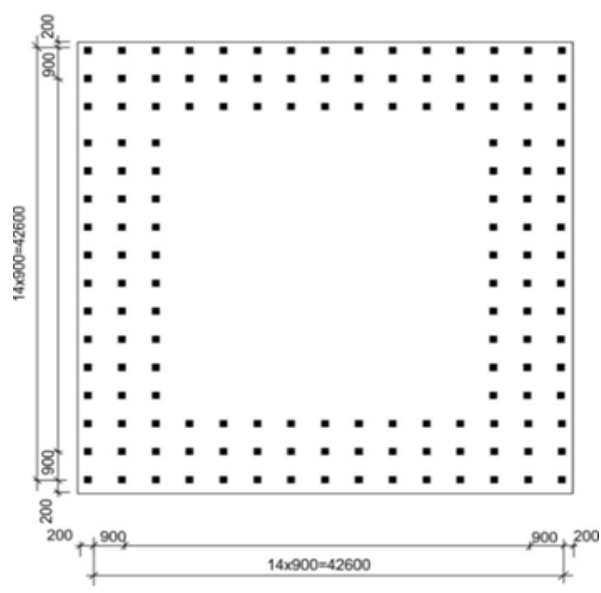

b)

Fig. 1. General view of the platform foundation (a) and pile placement plan (b)

Measurements of the parameters of dynamic vibrations were carried out using a complex with a six-component seismic platform. The complex is based on a digital control system based on a pulse-code modulation system and a computer designed to process input signals from differences in sources and issue commands to an analog control system along six platform axes.

Further, a theoretical analysis of the vibrations of the investigated object was carried out. When structures vibrate, serious factors affecting the nature of the vibrations are the conditions for embedding the foundation of the structure in the ground and the elastic properties of the foundation. The real conditions for embedding the foundation in the ground are difficult for mathematical analysis. In this regard, in the formulation of seismic effects, it is necessary to consider the problem of the interaction of the structure with the soil.

According to the accepted methodology in Construction Norms and Regulations, to calculate a structure for seismic resistance, he uses dynamic design schemes mainly to determine the periods and forms of natural vibrations. However, practice shows that the most significant difficulties are caused precisely by this part of the calculation in the associated choice of the calculation scheme. Dynamic calculations are more complicated than static ones. Therefore, the simplest types of design schemes are usually considered. Calculation schemes are not only designed to simplify the phenomenon under study; they, at the same time, emphasize the main characteristics on which it depends. If the main characteristics are quantitatively taken into account correctly enough, then the calculation results will correspond to reality with the necessary accuracy. 


\section{Methods}

To study the solution of problems, a differential equation is derived for the object's vibrations under consideration. To solve the differential equation and obtain the corresponding result, characteristic types of securing structures with pile foundations and design schemes were selected (Fig. 2, Fig. 3-5). The design scheme of the movement of a platform with a pile foundation under dynamic influences will be for three types of fastening a pile to a structure: hinged-supported ends of a pile to a building, rigid pinching of a building with a pile foundation, elastic pinching of a building with a pile foundation.
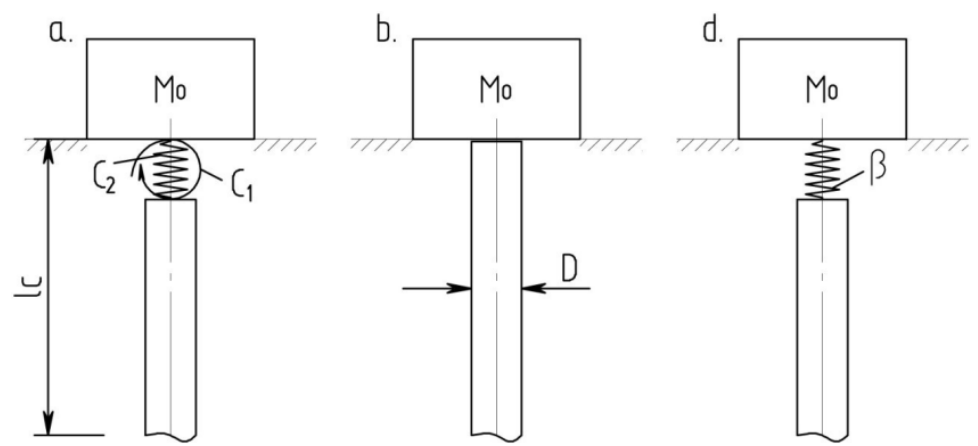

Fig. 2. Typical types of securing structures with pile foundations: a) combined elastic fastening; b) rigid fastening; d) elastic fastening.

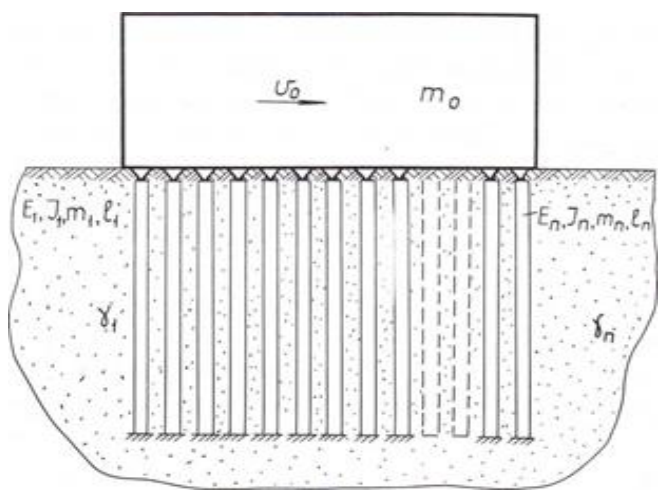

Fig. 3. Design scheme. Hinged pile ends to the building.

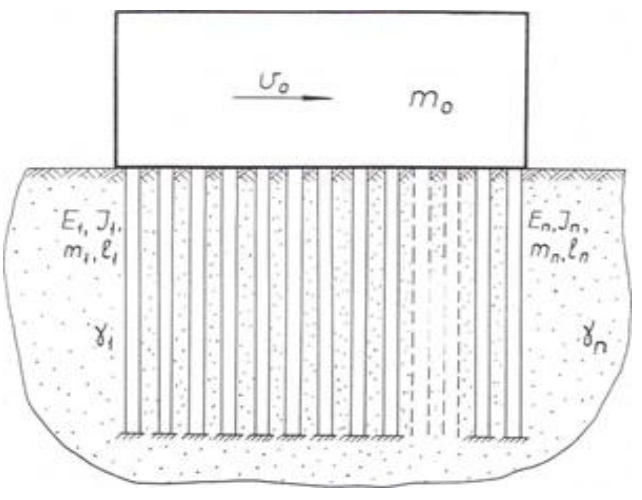

Fig. 4. Calculation scheme. Rigid anchorage of a building with pile foundations. 


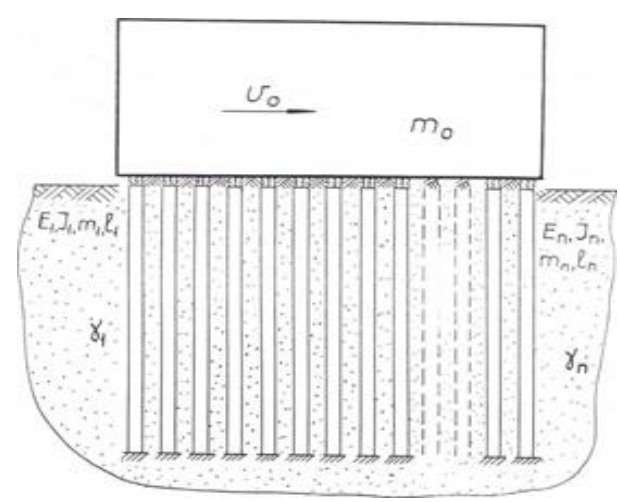

Fig. 5. Calculation scheme. Elastic crush building

Thus, the choice of the calculation scheme consists of analysing the studied process and quantitative assessment of the influence of individual factors on the calculation results. It should be noted that the minor factors taken into account in the design scheme cannot always clarify the calculation results, since in this case, it is necessary to have a large number of constant parameters, the specific values of which are often known only approximately. On the other hand, mathematical tabs become more complicated, which leads to additional operations and errors in calculations. It is desirable to have the design scheme as simple as possible. However, the adopted simplifications should be sufficiently substantiated and confirmed experimentally.

Here we will consider the transverse vibrations of the system of groups of piles elastic restrained with a rigid body at the level of overlaps and vibrations of groups of piles secured by hinged ends.

Consider, first of all, the transverse vibrations of the system of groups of elastic piles restrained with a rigid body at the floor level. (figure 5).

Let's designate $\vartheta_{n}(\mathrm{x}, \mathrm{t})$ is displacement of the $n$-pile, $\vartheta_{0}(t)$ is displacement of the floor mass. These displacements satisfy the following system of differential equations

$$
E_{n} I_{n} \frac{\partial^{4} \vartheta_{n}}{\partial x^{4}}+m \frac{\partial^{2} \vartheta_{n}}{\partial t^{2}}+\mu \frac{\partial \vartheta_{n}}{\partial t}+K \vartheta_{n}=0
$$

where $\mu$ is the coefficient of viscous friction, which characterizes the damping of vibrations during the interaction of the piles with the soil medium.

It is required to find a solution to the system of differential equations (1) that satisfies the following boundary conditions:

$$
E_{n} I_{n} \frac{\partial^{4} \vartheta_{n}}{\partial x^{4}}=K_{n}\left(\vartheta_{n}-\vartheta_{0}\right), \frac{\partial^{2} \vartheta_{n}}{\partial t^{2}}=0, \text { at } x=0 \vartheta_{n}=0, \frac{\partial \vartheta_{n}}{\partial x}=0 \text { at } x=l_{n}
$$

where $E_{n}$ is the modulus of elasticity; $I_{n}-$ is the moment of inertia; $m-$ is running weight of the pile; $K$ is the coefficient of lateral soil resistance; $K_{n}$ is the coefficient of stiffness of the connection of the mass (platform) with $n$ - pile.

The equation of motion of the masses is as follows: 


$$
m_{0} \frac{d^{2} \vartheta_{0}}{d t^{2}}=\sum_{n=1}^{N} K_{n}\left(\vartheta_{n}-\vartheta_{0}\right)+m_{n l} \omega_{0}^{2} \sin \omega_{0} t
$$

Where $l_{n}$ is length of $n$ - pile; $N$ is number of piles; $m_{0}$ is floor weight (platform); $m_{n l}$ is weight of a table with a pusher; $A_{n l}$ is seismic platform movement.

After some transformations, we can get the amplitude of oscillations of the floors in the form

$$
X_{0}=-\frac{\alpha_{1} j_{0}}{\alpha_{0} \tilde{\omega}_{0}^{2}+\sum_{n=1}^{N} \chi_{n} \frac{a_{n} \beta_{n} \lambda_{n}^{3}}{b_{n} \beta_{n}-\lambda_{n}^{3} \chi_{n} a_{n}}}
$$

The expression for determining the shear force for the $n$ - pile can be written in the following form:

$$
X_{0}^{\prime \prime \prime}=-\frac{\alpha_{1} j_{0} a_{n} \beta_{n} \lambda_{n}^{3}}{\left(b_{n} \beta_{n}-\lambda_{n}^{3} \chi_{n} a_{n}\right)\left(\alpha_{0} \tilde{\omega}_{0}^{2}+\sum_{n=1}^{N} \chi_{n} \frac{a_{n} \beta_{n} \lambda_{n}^{3}}{b_{n} \beta_{n}-\lambda_{n}^{3} \chi_{n} a_{n}}\right)}
$$

Fig. 6 and 7 show the amplitude-frequency characteristic and shearing force for different numbers of piles.

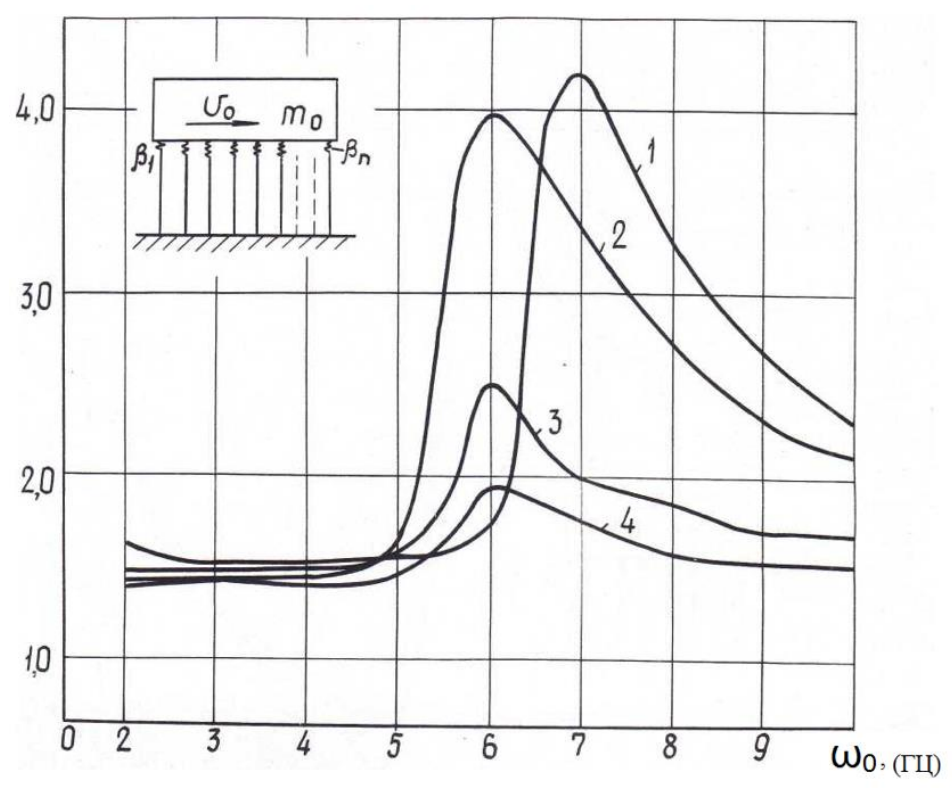

Fig. 6. Graph of changes in the amplitude-frequency characteristics of the system depending on the number of piles with elastic fastening: $1-N=144,2-N=120,3-N=50,4-N=30$ 


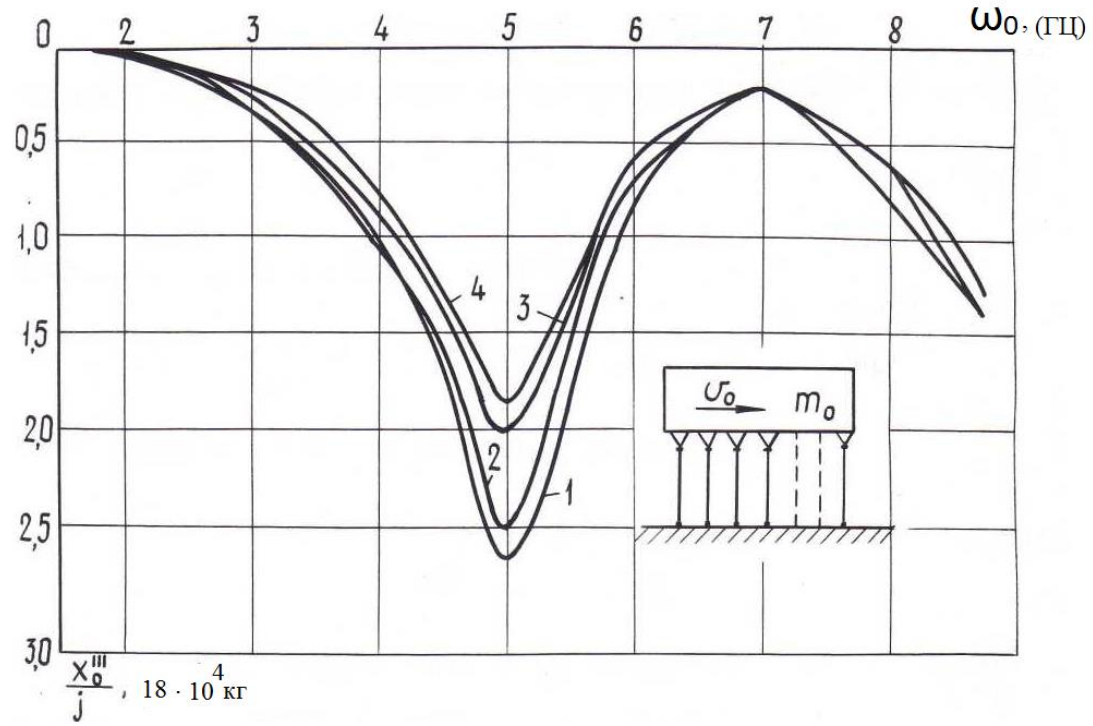

Fig. 7. Graph of the change in the value of the shearing force of the system versus the number of $1-N=144,2-N=120,3-N=50,4-N=30$

Next, we will consider the vibrations of groups of piles secured by hinged-supported ends (Figure 3).

The equation of motion of such a complex has the form:

$$
\frac{\partial^{4} \vartheta_{n}}{\partial \xi^{4}}+\frac{\partial^{2} \vartheta_{n}}{\partial \tau^{2}}+\gamma_{n} \vartheta_{n}=0
$$

Determine the maximum shear force in the support part of the piles

$$
X_{n}^{\prime \prime \prime}(0)=D_{n} \lambda_{n}^{3}=q_{n} \lambda_{n}^{3} X_{0}
$$

From here, after some transformations, we find the vibration amplitudes of the system

$$
X_{0}=-\frac{\alpha_{1} j_{0} \tilde{\omega}_{0}^{2}}{\alpha_{0} \tilde{\omega}_{0}^{2}+\sum_{n=1}^{N} \chi_{n} \lambda_{n}^{3} q_{n}}
$$

Figures 8 and 9 show the dependences of the quantities $\frac{X_{0}}{j_{0}}$ and $\frac{X_{0}^{\prime \prime \prime}}{j_{0}}$ the frequency for different values of the number of piles. 


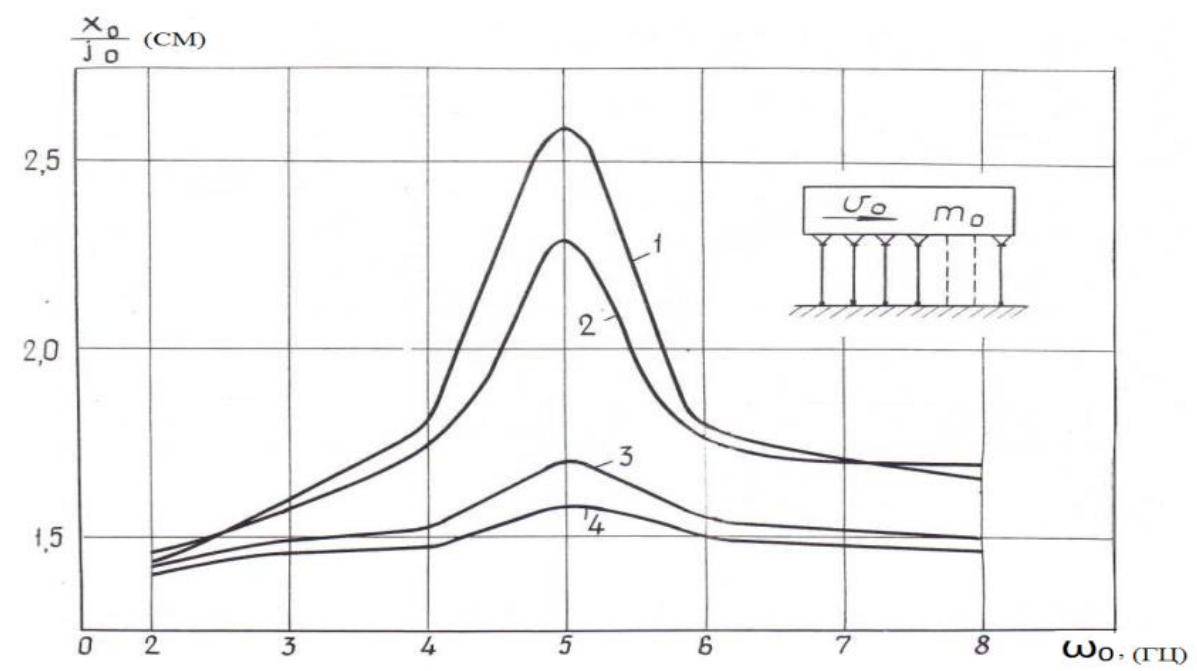

Fig. 8. Graph of changes in the amplitude-frequency characteristics of the system depending on the number of piles, with hinge-supported fastening. I- $N=144,2-N=120 ; 3-N=50,4-N=30$

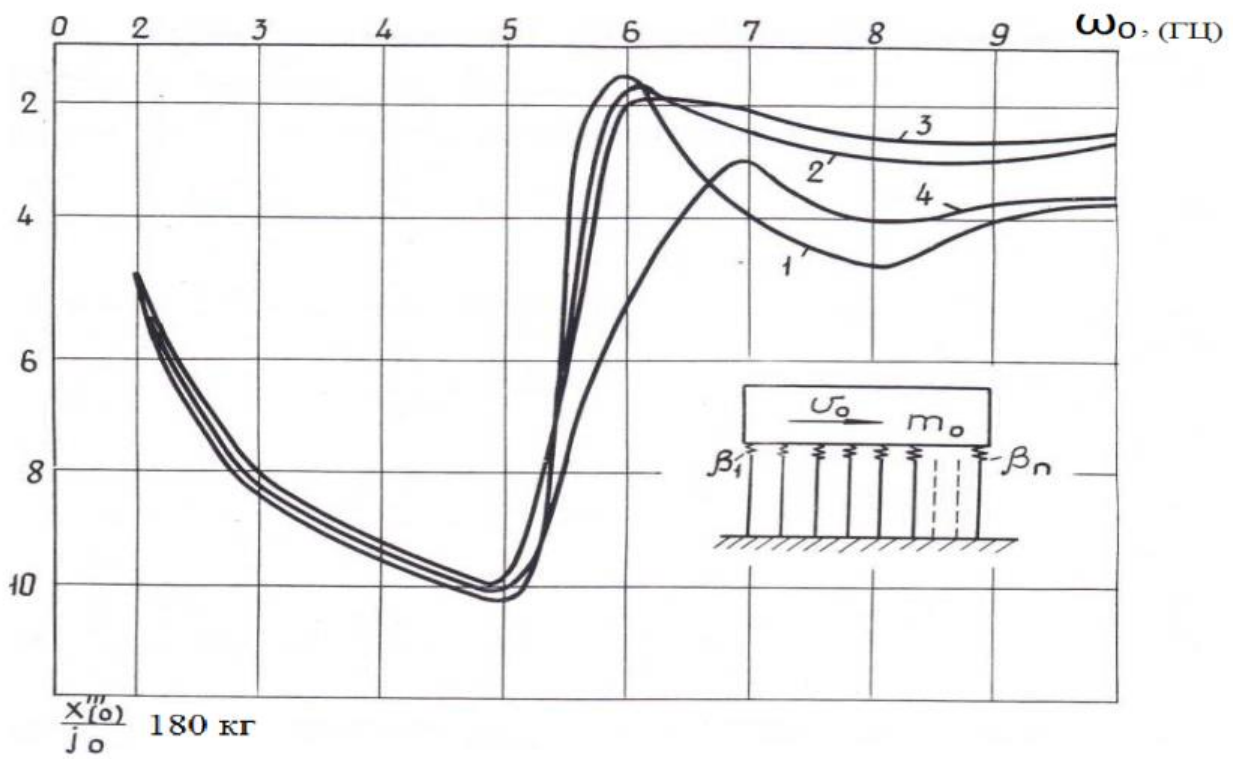

Fig. 9. Graph of the change in the shear force of the system versus the number of piles. I$N=144 ; 2-N=120 ; 3-N=50 ; 4-N=30$.

Now consider the rigid pinching of the pile with the platform and soil (Fig. 4). As in the previous calculations, after carrying out some operations, we define arbitrary constants based on the boundary conditions:

The expression for determining the shearing force at $\xi=0$ is as follows:

$$
X_{n}^{\prime \prime \prime}(0)=d_{n} \lambda_{n}^{3} X_{0}
$$

Continuing the calculations, we obtain the amplitude of system oscillations in the form 


$$
X_{0}=-\frac{\alpha_{1} j_{0} \tilde{\omega}_{0}^{2}}{\sum_{n=1}^{N} \chi_{n} \lambda_{n}^{3} d_{n}+\alpha_{0} \tilde{\omega}_{0}^{2}}
$$

In fig. 10 and 11 show the dependences of the quantities $\frac{X_{0}}{j_{0}}$ and $\frac{X_{0}^{\prime \prime \prime}}{j_{0}} \quad$ on the frequency for different values of the number of piles.

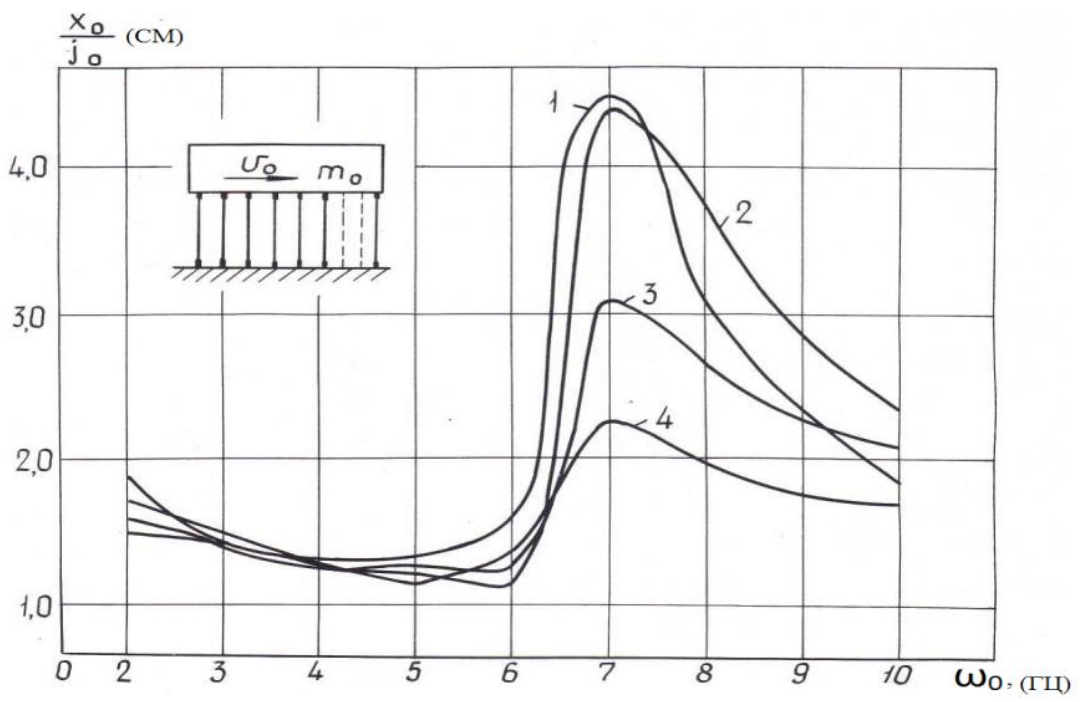

Fig. 10. Graph of changes in the amplitude-frequency characteristics of the system depending on the number of piles with rigid fastening. I $-N=144,2-N=120,3-N=50,4-N=30$.

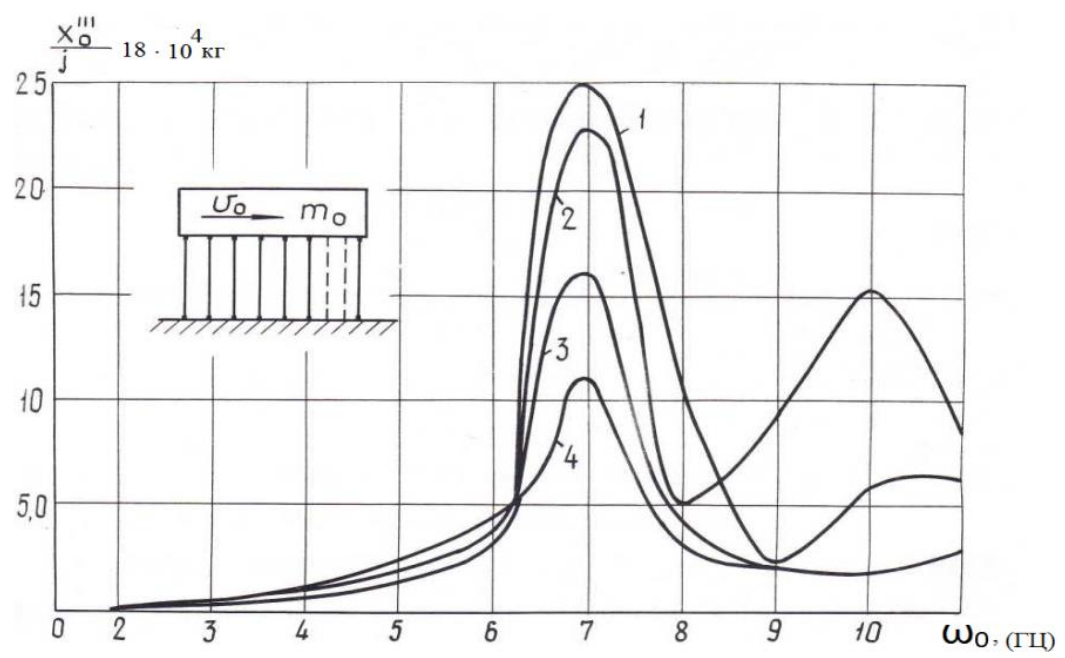

Fig.11. A graph of the change in the shear force value versus the number of piles. I $-N=144,2$ $N=120,3-N=50,4-N=30$. 


\section{Results and discussion}

Figure 6 shows the curves of the vibration amplitude of a platform fixed to piles without inertial elastic elements depending on the frequency for the following parameters: mass of the concentrated load (platform) $m_{0}=2000 \mathrm{t}$., running weight of a pile $m_{1}=2,2 \mathrm{t}$, elastic seal stiffness $\beta=2263 \mathrm{~kg} / \mathrm{sm}^{2}$, modulus of elasticity of concrete $E=2.7510^{5} \mathrm{~kg} / \mathrm{sm}^{2}$, pile moment of inertia $I_{1}=6.7510^{4} \mathrm{sm}^{4}$, soil bed coefficient $K=0.675 \mathrm{~kg} / \mathrm{sm}^{3}$, pile length $l=10 \mathrm{~m}$, seismic platform movement $A_{n л}=100 \mathrm{~mm}$, pusher table weight $m_{n л}=28 \mathrm{t}$, pile cross-section $30 \times 30 \mathrm{sm}$. It follows from these curves that with an increase in the number of piles, the amplitude of platform oscillation at low frequencies $0 \leq \omega_{0} \leq 5$, can be considered almost constant. Further, with increasing frequency $\left(\omega_{0} \geq 5\right)$, the oscillatory process occurs near the first resonance frequency, and the amplitude near the resonance is limited. With an increase in the number of piles, the value of the maximum amplitude increases and at the same time, can reach the value of the resonant frequency.

At $N \leq 120$ and further, resonance occurs at high frequencies. An increase in the maximum amplitude with an increase in the number of piles can be explained by the fact that an increase in the number of piles leads to an increase in the rigidity of the "platformpile" system. This indicates the possibility of a resonance phenomenon at high frequencies. Analysis of the curves for the amplitude of the shearing force (Fig. 7) shows that the magnitude of this force at frequencies $\omega_{0} \leq 6, N \leq 120$, practically does not depend on the number of piles and significantly depends on the frequency of external influences. The effect of the number of piles on the shear force is observed at high frequencies, starting with $\omega_{0} \geq 6$. In this case, the amplitude of this force, after reaching the maximum value $\left(\omega_{0}=6\right)$, increases slightly and remains practically constant. It is interesting to note that the platform displacement and shear force amplitudes reach their maximum values at the same frequency.

Figures 8 and 9 show similar curves when the platform is pivotally attached to the piles without considering the resistance to rotation. The curves show that the resonant frequencies move to the low frequency region, while they do not depend on the number of piles. The magnitude of the resonant amplitude of displacement in comparison with elastic pinching decreases by 1.5-2 times, and the magnitude of the amplitude of the shearing force for the selected parameters increases by several orders of magnitude.

The nature of the growth of shear forces near resonance frequencies differ significantly from each other. In fig. 10 and 11 show resonance curves in the case of rigid fastening, and the resistance forces on rotation, at the junction of the piles to the platform linearly depend on the angle of rotation of the pile section. The presence of such a force increases the rigidity of the system compared to the case of hinge-supported fastening. Calculations show that the amplitude of the shearing force for the selected parameters of the system increases by 8-10 times compared to the hinge pinning of the piles to the platform. In addition, the resonant frequency moves to the higher frequency region and a second resonant frequency appears for the shear force amplitudes. A comparison of the resonance curves for the displacement amplitudes and shear forces follows that the type of pile anchoring to the platform (structure) significantly affects the maximum amplitudes and the nature of these curves. The presence of elastic elements reduces the shear and rotation stiffness of the system, which makes it possible to reduce the magnitude of shearing forces at the joints by several orders of magnitude. Thus, choosing the stiffness of these elements, it is possible to 
carry out the process of damping (forces) arising in the sections of the pile anchoring to the platform.

\section{Conclusions}

1. Based on the results of theoretical studies, resonance curves were constructed for two types of fastening of the "structure-group of piles" system, including the resonance states of the system based on the experiments carried out.

2. A special technique was developed, and a series of field tests were carried out to determine the amplitude-frequency characteristics (AFC) of the "platform-group of piles" system; the regularity of resonance curves was established in a wide range of changes in the frequencies of the external exciting force.

3. It was found that, unlike buildings located on a strip foundation, the connection between the building and the soil foundation, which is a source of seismic impact, decreases here, which leads to a decrease in the intensity of the transmitted seismic load.

4. A dynamic model of the "platform-group of piles" system is proposed, which takes into account the influence of the number of piles, the method of their fastening to the platform, as well as the law of interaction with the surrounding soil on the vibration parameters of the system.

5. The results of theoretical studies show that when the coefficient of stiffness of the connection between piles and structures is $\mathrm{kg} / \mathrm{sm} 2$ the amplitude of displacements is closer than when to the experimental result at frequencies of $\mathrm{Hz}$. The discrepancy between the results of theoretical and experimental studies is about $20 \%$. Hence, we can conclude that the accepted design scheme of theoretical studies was chosen correctly, and it can be used to calculate pile systems for seismic effects with different amplitude-frequency characteristics.

6. The results of theoretical and experimental studies are confirmed by the reliability of the operation of structures on pile foundations under seismic and dynamic impacts.

\section{References}

1. Nurieva D.M. Calculation of pile foundations for seismic effects,-pp.43, Kazan, (2016)

2. Vatin N.I. Construction of pile foundations. St. Petersburg. Polytechnic University Press, pp. 225, (2012)

3. Buzrukov Z.S. Selection of the design scheme of the "flat frame-grillage-group of piles" system under dynamic load, UNIVERSIUM, № 12(81), pp. 86-926 Moscow, (2020)

4. Buzrukov Z.S. Features of the design of the foundations of high-rise buildings, taking into account soil conditions, "Bulletin of Science and Education", № 22 (100), pp.7986, Moscow, (2020)

5. Pedro Y. Interaction of elements of the "pile-grillage-building frame" system under seismic action, p.110, Moscow, (2000)

6. Buzrukov Z. and Khamrakulov A. Joint work of a flat frame and pile foundations under dynamic impacts, International Conference on Robotics and Mechantronics. IOP Conf. Series: Materials Science and Engineering 517, 011001, (2019)

7. Gorbunov M.I., Ilyichev V.A., Krutov V.I. Foundations, foundations and underground structures, pp. 153, Moscow, (1985)

8. Shvetsova G.I. Foundations and foundations, Handbook, M, Higher school, p. 382, (1991) 
9. Raximov A.M., Alimov X.L., To'xtaboev A.A. Mamadov B.A., Muminov K.K. Heat and Humidity Treatment of Concrete in Hot Climates, International Journal of Progressive Sciences and Technologies (IJPSAT), 24 № 1, pp. 312-319, (2020)

10. Raximov A.M., Alimov X.L., Jurayev B.G. Strength development of concrete on Sraining cemtnt.5 th th International conference Recent Nrends in Yumanities, Technology, Manegement and Social Development, RTHTMS, (2020)

11. Alimov H.L. Determination of the dynamic characteristics of pile foundations of structures. pp. 20, Tashkent, (1991) 